\title{
Progression from Acute Kidney Injury to Chronic Kidney Disease: Clinical and Experimental Insights and Queries
}

\author{
Richard A. Zager \\ Department of Medicine, University of Washington, and Fred Hutchinson Cancer Research Center, Seattle, Wash., USA
}

\section{Key Words}

Acute kidney disease $\cdot$ Acute uremia $\cdot$ Azotemia $\cdot$ Bilateral ischemia . Chronic kidney disease - Unilateral ischemia

\begin{abstract}
There is an increasing number of clinical studies suggesting that acute kidney injury (AKI) can be complicated by the onset of progressive renal disease. Indeed, given the frequency of AKI in hospitalized patients, it could potentially be a leading cause of, or contributor to, end-stage renal disease. Insights into the natural history of AKI and potential mechanisms for disease progression can be gleaned from experimental studies. Although such studies underscore the principle that AKI can 'heal with defects', whether ongoing renal disease develops remains a subject of debate. Indeed, in the aftermath of AKI, a variety of secondary renal protective pathways are activated, which may retard or prevent severe chronic kidney disease. Furthermore, the onset of acute uremia per se may exert surprisingly potent renal protective effects. The purpose of this brief report is to review some of the clinical and experimental data that deal with these complex issues.

(c) 2014 S. Karger AG, Basel
\end{abstract}

(c) 2014 S. Karger AG, Basel

$1660-2110 / 14 / 1274-0046 \$ 39.50 / 0$

\section{Introduction}

Although severe AKI has existed throughout history, it was not until the introduction of acute dialysis $\sim 50$ years ago that the natural course of AKI could be defined. This was made possible because dialysis allows for sufficient time for renal recovery to occur and for possible subsequent disease progression to develop. Early investigators reported that 'the vast majority of patients who survive achieve clinically normal renal function despite frequent subclinical and histological defects' [reviewed in 1]. These included $20-40 \%$ reductions in GFR, urinary concentration, and acidification defects, and histologic evidence of interstitial fibrosis. It was also suggested that in a small number $(<5 \%)$ of patients in whom renal recovery is incomplete, a secondary regression of renal function may be observed' [1].

During the past 10 years, there has been increasing interest in, and awareness of, the prospect that AKI can indeed progress to chronic kidney disease (CKD). Howev-

Targeting Recovery from Acute Kidney Injury: Round Table Conference at the 19th International Conference on Continuous Renal Replacement Therapies (Manchester Grand Hyatt, San Diego, Calif., USA, March 2-3, 2014).

\section{KARGER}

E-Mail karger@karger.com www.karger.com/nec
Richard A. Zager, MD

Fred Hutchinson Cancer Research Center

1100 Fairview Avenue N, Room D2-190

Seattle, WA 98109 (USA)

E-Mail dzager@fhcrc.org 
er, it still remains uncertain as to the frequency of this transition and its underlying mechanisms. One critical study which addressed this issue came from Lo et al. [2]. These investigators followed the course of 703 patients with AKI who required acute renal replacement therapy. Of these individuals, 508 survived, allowing for their subsequent renal course to be discerned. Based on that study, it was suggested that there was a 28 -fold increased risk of developing either stage 4 or stage 5 renal disease [2]. However, it would appear that, in the vast majority of cases, this outcome was apparent within 3 months of the onset of AKI. Indeed, beyond that time point, the KaplanMeier slope for renal disease onset or progression was essentially identical to that observed in a cohort of hospitalized controls without AKI [2]. Thus, it remains unclear as to how many patients actually developed progressive disease above and beyond the early renal dysfunction/'healing with a defect', as noted by early investigators of this field [1].

\section{Can Experimental AKI Models Provide Insights into the Course of AKI and Possible Disease Progression?}

In order to better define the natural history of AKI, investigators have turned to experimental models. However, an inherent limitation of these studies is that the severity of the initial renal insult must be severe enough to induce substantial tubular damage, but yet mild enough to prevent early animal death from acute uremia. This is because of the technical difficulties inherent in supporting experimental animals with dialytic therapy. Despite this limitation, important insights have been gleaned. In this regard, seminal studies by Basile et al. [3, 4] have employed the bilateral renal ischemia model to explore these issues. One such study employed a rat model of 60 -min bilateral renal ischemia, with subsequent renal functional and histologic assessments performed over the ensuing 40 weeks [3]. Noteworthy in this regard is that 40 weeks represents $\sim 25 \%$ of a normal rat life span, clearly long enough to ascertain the natural course of AKI. In those studies, severe initial AKI was induced with early postischemic reductions in glomerular filtration rate (GFR) of approximately $80 \%$. However, renal functional recovery promptly ensued, and over the ensuing 40 weeks, only a 10\% GFR reduction (nonsignificant) below normal values (in sham-operated rats) was observed. Of note, however, is that renal histology demonstrated modest interstitial fibrosis and an approximate $35 \%$ reduction in the density of peritubular capil- laries. The latter was posited to contribute to ongoing ischemic/hypoxic tissue damage. Thus, while these workers demonstrated that ischemic AKI did, indeed, produce permanent renal damage after 40 weeks, severe progressive CKD did not ensue. Hence, these experimental findings are consistent with the above-noted clinical conclusion: that 'the vast majority of AKI results in clinically normal renal function despite frequent subclinical and histological defects' [1].

Nath et al. [5] have also evaluated the issue of longterm AKI outcomes using a model of repetitive nephrotoxic insults. These workers employed weekly injections of hypertonic glycerol into the hind limbs of rats, inducing repetitive bouts of muscle necrosis and myohemoglobinuria. After a single dose of glycerol, severe AKI was produced. They then administered glycerol on a weekly basis for 6 months, and at the end of these injections, an approximate one-third loss of GFR was observed. However, no loss of renal parenchymal mass (renal weight) and only minimal proteinuria developed. Furthermore, it is notable that, in that study, the rats continued to lose body weight. Thus, in light of the renal mass preservation, it is not clear whether cachexia, which is known to reduce GFR, contributed to the observed one-third GFR decline. One potential explanation for this seemingly benign renal outcome despite severe repetitive bouts of rhabdomyolysis is that AKI induces a variety of cytoprotective mechanisms (e.g. an upregulation of heme oxygenase-1) [6], which could serve to mitigate ongoing renal damage. These considerations notwithstanding, the data would certainly seem to challenge the accepted notion that repetitive bouts of AKI culminate in progressive renal disease.

\section{Unilateral Ischemic Renal Injury Recapitulates Progression from AKI to End-Stage Renal Disease}

Sixty years ago, Koletsky [7] made the observation that 60-min unilateral ischemia results in severe and progressive postischemic injury, which culminates in end-stage kidney disease. This stands in sharp contrast to the results of Basile et al. [3, 4], discussed above, whereby $60 \mathrm{~min}$ of bilateral ischemic injury failed to produce sustained renal failure. Based on these divergent results, we recently tested the hypothesis that unilateral, but not bilateral, ischemic renal injury induces progressive renal disease. Therefore, we induced $30 \mathrm{~min}$ of unilateral ischemia in a mouse model, and severe progressive renal failure ensued $[8,9]$. Thus, within just $2-3$ weeks after unilateral isch- 
emia, severe interstitial inflammation, fibrosis, tubular dropout, and a $67 \%$ loss of renal mass were observed. Conversely, when bilateral ischemia was induced, renal mass was well preserved. To further illustrate this point, we induced 30-min unilateral (left) kidney ischemia combined with progressive right kidney ischemic damage (renal artery occlusion for 0-20 min). Thus, we were able to induce graded azotemia, which was assessed $24 \mathrm{~h}$ after surgery [10]. The key finding of that study was that the greater the length of the contralateral ischemic damage, the greater was the initial azotemia, and the lesser the amount of left kidney damage, which was assessed 2 weeks later. Thus, unilateral ischemia produced endstage kidney disease, but this could be almost completely prevented by inducing contralateral ischemic damage. Stated differently, the greater the degree of initial azotemia (24-hour blood urea nitrogen levels), the lesser the degree of renal parenchymal loss during the $30 \mathrm{~min}$ following renal ischemia (depicted in fig. 1).

\section{The State of Uremia Can Confer Cytoprotective Effects}

The strong correlation between renal protection and the initial severity of azotemia in the above study raised an intriguing hypothesis: that acute uremia can exert cytoprotective and anti-inflammatory effects, and that this 'uremic brake' prevents renal disease progression. A series of studies from our laboratory provides direct experimental support for this concept [11,12]. First, when unilateral ischemia was induced in the presence of uremia (by damaging the contralateral kidney), a marked suppression of proinflammatory/profibrotic cytokines and a marked upregulation of cytoprotective proteins (interleukin-10 and heme oxygenase-1) were observed. Hence, the balance between pro- versus anti-inflammatory molecules appeared to be tilted towards the antiinflammatory state. Second, when mice with severe renal failure underwent peritoneal dialysis, addition of that dialysate to cultured tubular cells conferred a cytoprotective/anti-inflammatory state. Third, when human urine samples were added to freshly isolated mouse proximal tubules (creating experimental uremia in vitro), the tubules became resistant to hypoxic attacks. Fourth, the creation of experimental uremia in mice in the presence of normal kidneys (by performing peritoneal dialysis using normal human urine) protected the kidneys from subsequent ischemic damage [13]. In composite, these studies provide a potential explanation for why unilat-

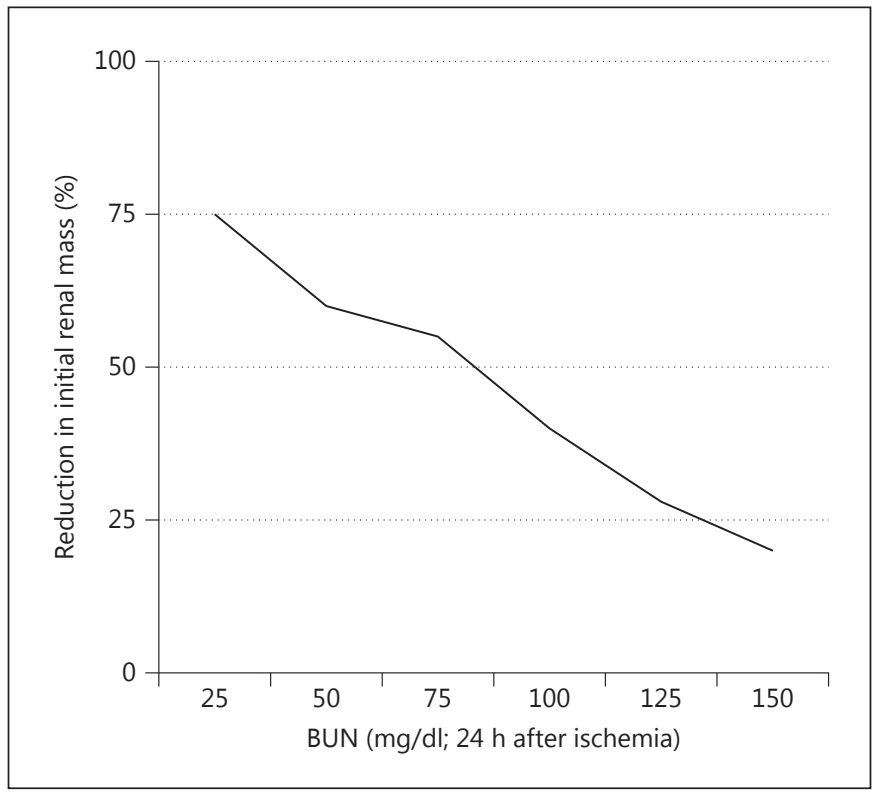

Fig. 1. The relationship between the severity of initial azotemia and the ultimate loss of postischemic renal parenchymal mass. Thirty minutes of ischemia was imposed on the left kidney with variable degrees of ischemia (0-20 min) imposed on the right kidney. As expected, this produced variable degrees of azotemia (assessed $24 \mathrm{~h}$ after surgery). The greater the degree of initial azotemia (assessed $24 \mathrm{~h}$ after $30 \mathrm{~min}$ of left ischemia $+0-20 \mathrm{~min}$ of right ischemia), the lesser the percent loss of left renal mass (assessed by renal weight) 2 weeks after surgery. $\mathrm{BUN}=$ Blood urea nitrogen.

eral ischemic injury (i.e., in the absence of uremia), but not bilateral ischemic injury (in the presence of uremia), leads to progressive renal disease. The key question is what component of the uremic milieu is responsible for the observed cytoprotective/anti-inflammatory state. To date, it appears that it is a low-molecular-weight, heatlabile substance that resists freeze-thaw cycles. Clearly, if this substance (or substances) could be identified, novel prophylactic and therapeutic opportunities might result.

\section{Potential Therapeutic Strategies for Preventing AKI Progression to CKD}

Although the clinical relevance of the unilateral ischemia model may be questioned (given that AKI is typically a bilateral disease), it does provide a unique opportunity to study pathways of disease progression after AKI. For example, Bonventre's group [14], studying this mod- 
el, has proposed that progression is mediated via proximal tubule cell cycle arrest at the G2/M phase, which results in the generation of profibrotic factors, including cytokines, growth factors, and matrix proteins. Conversely, this block was not present with bilateral ischemic damage. Alternatively, we have demonstrated that unilateral renal ischemia leads to a marked remodeling of chromatin at proinflammatory genes [9], which may contribute to unbridled gene activation, and presumably ongoing renal inflammation and fibrosis. The importance of intrarenal inflammation in this model was underscored by our observation that high-dose glucocorticoid therapy attenuated (but did not eliminate) progressive postischemic renal disease [8].

As noted previously, Basile et al. [3, 4] have provided novel insights that one potential reason for postischemic renal disease progression in rodents is the loss of peritubular capillaries, a process that presumably induces ongoing hypoxic tissue damage. These observations led us to question whether ongoing tissue hypoxia might be exacerbated by endothelin (ET)-1-mediated renal vasoconstriction. To this end, mice were subjected to $30 \mathrm{~min}$ of unilateral renal ischemia [15]. ET-1 mRNA and protein levels, as well as the mRNA for ET-1A and -1B receptors (which mediate vasoconstriction and vasodilation, respectively), were assessed $24 \mathrm{~h}$ or 2 weeks later. The impacts of potent ETA or ETB receptor-specific antagonists (atrasentan and BQ-788, respectively) on postischemic disease progression were also assessed. Unilateral ischemia caused massive upregulation of both renal cortical ET-1 mRNA and ET-1 protein levels ( 10- and 50-fold, respectively). Furthermore, parallel increases in ETA, but not the ETB, receptor mRNA, was also observed. A chromatin immunoprecipitation assay revealed extensive 'gene-activating' histone remodeling (methylation, acetylation, and H2A.Z enrichment) and increased RNA polymerase II binding at the ET-1 gene, thereby providing a potential explanation for ET-1 overexpression. The functional significance of ET-1 overexpression was indicated by observations that the ETA receptor blocker atrasentan, administered either before and after ischemia, or started $24 \mathrm{~h}$ after ischemia, conferred marked protective effects, which preserved renal mass. That ETA blockade also lowered renal tissue lactate levels suggested that its protective action was mediated, at least in part, by improvements in renal oxygen delivery. Conversely, ETB receptor blockade was without a beneficial effect. Based on these studies, it would appear that ischemic renal injury evokes progressive activation of the ET-1 gene, possibly by induction of 'gene-activating' histone remodeling at this site.
Furthermore, these studies provide the first evidence that ET-1 can play a critical role in postischemic AKI progression to CKD, at least in the unilateral ischemia model. Thus, even when AKI initiates renal disease progression, it is clearly not inevitable.

\section{Conclusions}

It has long been recognized that AKI has the capacity to initiate CKD. However, the frequency of disease progression remains a subject of considerable debate. Much of the clinical literature would suggest that renal disease following AKI may simply be a result of 'healing defects' rather than true progression. The experimental literature would appear to support this conclusion given that severe bilateral ischemic renal injury or recurrent nephrotoxic AKI has not been demonstrated to lead to severe progressive, or end-stage, kidney disease. Multiple factors undoubtedly impact whether or not disease progression results, e.g., whether or not preexisting renal disease exists, patient age, associated vascular disease, and whether or not recurrent bouts of injury occur. But if disease progression does result, it is clearly not inevitable. For example, selected mechanistic pathways can potentially be blocked (e.g., ET-1 overexpression or renal inflammation). Finally, if, indeed, uremic cytoprotectant/anti-inflammatory compounds truly exist, and if they can be identified and clinically deployed, then completely new pharmacologic approaches to prevent AKI induction and progression may emerge.

\section{Acknowledgment}

This work was supported by research grants from the National Institutes of Health (DK38432 and DK68520).
References
1 Brezis M, Rosen S, Epstein FH: Acute renal failure; in Brenner BM, Rector F (eds): The Kidney. Philadelphia, Saunders, 1986, chapt, 19, pp 735-799.
2 Lo LJ, Go AS, Chertow GM, McCulloch CE, Fan D, Ordoñez JD, Hsu CY: Dialysis-requir- ing acute renal failure increases the risk of progressive chronic kidney disease. Kidney Int 2009;76:893-899.
-3 Basile DP, Donohoe D, Roethe K, Osborn JL: Renal ischemic injury results in permanent damage to peritubular capillaries and influ- ences long-term function. Am J Physiol Renal Physiol 2001;281:F887-F899. 
4 Basile DP, Friedrich JL, Spahic J, Knipe N, Mang H, Leonard EC, Changizi-Ashtiyani S, Bacallao RL, Molitoris BA, Sutton TA: Impaired endothelial proliferation and mesenchymal transition contribute to vascular rarefaction following acute kidney injury. Am J Physiol Renal Physiol 2011;300:F721-F733.

$\checkmark 5$ Nath KA, Croatt AJ, Haggard JJ, Grande JP: Renal response to repetitive exposure to heme proteins: chronic injury induced by an acute insult. Kidney Int 2000;57:2423-2433.

6 Nath KA: Heme oxygenase-1: a provenance for cytoprotective pathways in the kidney and other tissues. Kidney Int 2006;70:432-443.

7 Koletsky S: Effects of temporary interruption of renal circulation in rats. AMA Arch Pathol 1954;58:592-603.
Zager RA, Johnson AC, Becker K: Acute unilateral ischemic renal injury induces progressive renal inflammation, lipid accumulation, histone modification, and 'end-stage' kidney disease. Am J Physiol Renal Physiol 2011; 301:F1334-F1345.

-9 Zager RA, Johnson AC: Renal ischemia-reperfusion injury upregulates histone-modifying enzyme systems and alters histone expression at proinflammatory/profibrotic genes. Am J Physiol Renal Physiol 2009;296:F1032F1041.

10 Zager RA, Johnson AC, Becker K: Post-ischemic azotemia as a partial 'brake', slowing progressive kidney disease. Nephrol Dial Transplant 2013;28:1455-1462.

11 Zager RA: Uremia induces proximal tubular cytoresistance and heme oxygenase-1 expression in the absence of acute kidney injury. Am J Physiol Renal Physiol 2009;296:F362-F368.
2 Zager RA, Johnson AC, Lund S: Uremia impacts renal inflammatory cytokine gene expression in the setting of experimental acute kidney injury. Am J Physiol Renal Physiol 2009;297:F961-F970.

13 Zager RA, Baltes LA, Sharma HM, Jurkowitz MS: Responses of the ischemic acute renal failure kidney to additional ischemic events. Kidney Int 1984;26:689-700.

14 Yang L, Besschetnova TY, Brooks CR, Shah JV, Bonventre JV: Epithelial cell cycle arrest in G2/M mediates kidney fibrosis after injury. Nat Med 2010;16:535-543.

15 Zager RA, Johnson AC, Andress D, Becker K: Progressive endothelin-1 gene activation initiates chronic/end-stage renal disease following experimental ischemic/reperfusion injury. Kidney Int 2013;84:703-712. 